\title{
473931 - LUMBAR INTRATHECAL ADMINISTRATION OF THE QUATERNARY LIDOCAINE DERIVATIVE, QX-314, PRODUCES NEUROTOXICITY IN MICE
}

\author{
Stephan Schwarz, M.D., Ph.D., FRCPC ${ }^{1}$, Helen Cheung, B.Sc. ${ }^{1}$, Craig Ries, M.D., \\ FRCPC, Ph.D. ${ }^{1}$, Sang Lee, M.D. ${ }^{2}$, Jimmy Wang, B.Sc. ${ }^{1}$, Bernard MacLeod, M.D., \\ FRCPC $^{1}$
}

1. Department of Anesthesiology, Pharmacology \& Therapeutics, The University of British Columbia, Vancouver, BC, Canada

2. Department of Anesthesiology and Pain Medicine, Catholic University Medical College, Daejon, South Korea

Introduction: The quaternary lidocaine derivative, QX-314, has traditionally been considered to be devoid of clinically useful local anesthetic activity. However, we recently found that QX-314, administered peripherally, concentration-dependently and reversibly produces long-lasting local anesthesia with a slow onset in animal models in vivo (1). The goal of the present study was to determine whether QX-314 would have similar properties when administered as a spinal anesthetic.

Methods: With approval by the institutional Animal Care Committee, we conducted a randomized, double controlled, blinded study with female CD-1 mice naïve to drug administration, preceded by a pilot dose-finding phase for QX-314. Animals allocated to the treatment group were injected with QX-314 (0.5, 3, 5, or $10 \mathrm{mM})$ into the lumbar intrathecal space (L5/L6; volume, $2 \mu \mathrm{l}$; each dose, $n=6$ ) under sevoflurane anesthesia. Normal saline and lidocaine (70 mM [ 2\%]) served as negative (placebo) and positive controls, respectively (each group, $n=12$ ). Following emergence, animals were observed for up to $3 \mathrm{hr}$ and tested for reversible hind limb paresis and tail flaccidity as endpoints of lumbosacral subarachnoidal motor blockade. In addition, we tested animals for sensory blockade by their response to tail clamp application, and for their ability to hang on to an inverted mesh with their hind limbs as a supplemental assay for motor blockade. Lastly, we observed animals for signs of neurotoxicity and other adverse events. Data were analyzed with Fisher's exact test and ANOVA as appropriate; $\mathrm{P}<0.05$.

Results: None of the 12 animals injected with saline and 11/12 (92\%) of animals injected with lidocaine had evidence of reversible lumbosacral subarachnoidal motor blockade ( $\mathrm{P}$ $<0.01$ ). Animals injected with QX-314 concentration-dependently exhibited signs of neurotoxicity and death. In the pilot experiments, 2/2 animals injected with $30 \mathrm{mM}$ QX314 showed evidence of severe sustained distress (including vocalization, scratching, flinching, and twitching); one died. Consequently, we limited the QX-314 concentrations for the randomized blinded study to a maximum of $10 \mathrm{mM}$. At $10 \mathrm{mM}$, QX-314 produced sensory blockade in 4/6 animals (67\%) and motor blockade in 3/6 animals (50\%; P < 0.05 compared to saline). However, 6/6 mice (100\%) at $5 \mathrm{mM} \mathrm{QX-314} \mathrm{and} \mathrm{5/6} \mathrm{(83 \% )} \mathrm{at}$ $10 \mathrm{mM}$ exhibited severe agitation (sustained circling, jumping, and/or vocalization), irritation (scratching, flinching, shaking, and/or writhing), and/or persistent loss of righting reflex; 1/6 animals at $5 \mathrm{mM}$ (17\%) and 2/6 at $10 \mathrm{mM}$ (33\%) died. We observed no local anesthetic effects without evidence of neurotoxicity in any animal injected with 
QX-314 (0.5-10 mM). None of the animals injected with saline showed signs of neurotoxicity. One animal injected with lidocaine displayed irritable behaviour; all others (11/12 or 92\%) exhibited no evidence indicative of neurotoxicity.

Discussion: QX-314 administered in the lumbar intrathecal space concentrationdependently produces neurotoxicity and death in mice, at lower concentrations than those associated with local anesthetic effects. These findings are in contrast to those with lidocaine and indicate that QX-314 is unlikely to be a suitable candidate for spinal anesthesia in humans.

References: 1. Anesthesiology 2007 107: 305-311 